\title{
Clarithromycin Induced Psychosis in an AIDS Patient Receiving Treatment for Disseminated Mycobacterium avium Intracellulare Infection
}

\author{
Sneha Jacob ${ }^{1 *}$, Jason Breig ${ }^{2}$ and Theodore Chou ${ }^{2}$ \\ ${ }^{1}$ Robert Wood Johnson University Hospital, New Brunswick, NJ, USA \\ ${ }^{2}$ MSIII, Rutgers University, Robert Wood Johnson Medical School, Piscataway, NJ, USA
}

\begin{abstract}
Here we present the case of a man who was admitted to the hospital with an extremely low CD4 count and vague symptoms suggestive of an opportunistic infection, who subsequently developed a significant psychosis following standard treatment for Mycobacterium avium intracellulare. The patient was a 48 year old man who presented to the hospital with acute onset altered mental status, diarrhea, and generalized weakness. He had positive blood cultures for Mycobacterium avium intracellulare and was given clarithromycin, ethambutol, and rifabutin. The patient developed an acute change in mental status manifested by paranoid delusions, disorganized though processes, and perseverations which resolved after substitution of azithromycin for clarithromycin. Although the differential diagnosis of acute altered mental status in an AIDS patient is quite broad, we recommend that clinicians consider clarithromycin as a possible cause of psychosis and mania. Azithromycin should be considered as an alternative treatment in patients exhibiting such symptoms after initiation of clarithromycin.
\end{abstract}

Keywords: Clarithromycin; AIDS; HIV; Psychosis; Mania; Mycobacterium avium; Altered mental status

\section{Introduction}

With the advent of antiretroviral therapy and prophylaxis/ treatment of opportunistic infections in individuals with Human Immunodeficiency Virus (HIV) and Acquired Immune Deficiency Syndrome (AIDS) there has been a significant decrease in the incidence of opportunistic infections over the past 30 years [1]. While these therapies have certainly increased the quality of life and decreased the morbidity and mortality of for patients diagnosed with HIV/ AIDS, they have brought with them unique adverse effect profiles that medical professionals must be aware of when treating their patients. Here we present the case of a man who was admitted to the hospital with an extremely low CD4 count and vague symptoms suggestive of an opportunistic infection, who subsequently developed a significant psychosis following standard treatment for Mycobacterium avium intracellulare.

\section{Case Presentation}

A 48 year old Caucasian gentleman with a past medical history significant for newly diagnosed HIV/AIDS (CD4 count $=6$ cells $/ \mathrm{mm}^{3}$ and HIV viral load $>1$ million copies in December 2013) presented to the hospital with acute onset altered mental status, diarrhea, and generalized weakness. The patient was previously hospitalized in December 2013 for an episode of weakness and diarrhea; however at that time he did not have any change in mental status. On the first hospital admission in December he was diagnosed with HIV/AIDS. Blood cultures drawn at that time later became positive for Mycobacterium avium intracellulare (MAI). The patient was discharged and followed up with an HIV specialist in the outpatient setting where pneumocystis pneumonia prophylaxis with Trimethoprim-Sulfamethoxazole was started. As the patient was uninsured the case manager initiated an application for the AIDS Drug Distribution Program (ADDP) in anticipation of the patient starting antiretroviral therapy as soon as it was approved.

The patient was reported to be in his usual state of health until the day of admission when his mother found him lying in his own feces and unable to move secondary to weakness. His mother states that he was responsive but confused with delayed speech and ambiguous responses to questions. She noted that he was aware that something was wrong but was unable to communicate his thoughts. The patient was not capable of ambulating due to weakness and had difficulty moving his extremities. He was able to remember the events leading up to the morning of admission but did not remember soiling himself or any events precipitating the hospitalization. The patient reported a recent onset of diarrhea following the initiation of TrimethoprimSulfamethoxazole and two episodes of vomiting during the night before admission. He also complained of generalized fatigue. The patient denied having any recent fevers, chills, night sweats, nausea, chest pain, shortness of breath, wheezing, headache, blurry vision, tinnitus, or any episodes of syncope. He denied any history of seizure like activity and any recent sick contacts. Further review of systems were negative.

The patient's past medical history includes recent diagnosis of HIV/AIDS and tachycardia with a heart rate between 90 and 110 . He denies any past surgical history. The patient denied any known drug allergies and on admission was taking metoprolol succinate 25 $\mathrm{mg}$ by mouth daily and trimethoprim-sulfamethoxazole $160 \mathrm{mg}$ $800 \mathrm{mg}$ by mouth daily. The patient's family history is significant for hypertension, hyperlipidemia, and coronary artery disease. The patient was unemployed and reports past employment as a truck driver. The patient denied any history of tobacco use, alcohol consumption, intravenous drug use, or any other use of illicit drugs. He reported being sexually active with multiple commercial sex workers in the past with inconsistent condom use. His last sexual contact was six months prior to admission with his present girlfriend who reported a history of commercial sex work.

On physical examination the patient was 73 inches tall and weighed 205 pounds. He was tachycardic with a heart rate of 136 On admission, his temperature and respiratory rate were within

*Corresponding author: Sneha Jacob, Robert Wood Johnson University Hospital, New Brunswick, NJ, 277 George Street, New Brunswick, NJ 08901, USA, Tel: 732 235-6724; Fax: 732-235-6726; E-mail: jacobse@rwjms.rutgers.edu

Received February 25, 2014; Accepted March 31, 2014; Published April 12, 2014

Citation: Jacob S, Breig J, Chou T (2014) Clarithromycin Induced Psychosis in an AIDS Patient Receiving Treatment for Disseminated Mycobacterium avium Intracellulare Infection. J AIDS Clin Res 5: 297. doi:10.4172/2155-6113.1000297

Copyright: ( 2014 Jacob S, et al. This is an open-access article distributed under the terms of the Creative Commons Attribution License, which permits unrestricted use, distribution, and reproduction in any medium, provided the original author and source are credited. 
normal limits. He appeared pale and looked older than his stated age. A cardiovascular exam revealed tachycardia, with normal S1/S2 and no associated murmurs, rubs, or gallops, lifts, heaves, or thrills. His lungs were clear to auscultation bilaterally with no wheezes, rales, or rhonchi appreciated. Abdominal exam revealed positive bowel sounds with a soft, non-tender, non-distended abdomen. His extremities were warm and well perfused with no clubbing, cyanosis, or edema. Neurological exam revealed no focal deficits and 5/5 motor strength noted bilaterally in both upper and lower extremities. He was awake and oriented to person, place, and time and exhibited delayed verbal responses to questioning. The patient's mental status exam was positive for circumstantial thought process with loose associations, impaired recent memory and poor insight without exhibiting any manic or psychotic features. An admission non-contrast cat scan of the head demonstrated ventriculomegaly. An MRI confirmed moderate ventriculomegaly but not did not otherwise show any suspicious CNS lesions. The admission chest $\mathrm{x}$-ray revealed no acute cardiopulmonary pathology. The patient's labs showed a white blood cell count of 3,900 cells/ $\mu \mathrm{L}$ with a neutrophilia of $88.5 \%$. His hemoglobin was slightly decreased at $11.6 \mathrm{~g} / \mathrm{dL}$. The patient's labs also showed mild hyponatremia $(134 \mathrm{mEq} / \mathrm{L})$, mild hyperkalemia (5.3 $\mathrm{mEq} / \mathrm{L})$, mild hypocalcemia $(8.0 \mathrm{mg} / \mathrm{dL})$, hypoalbuminemia $(2.5$ $\mathrm{g} / \mathrm{dL})$, and hypomagnesemia $(1.7 \mathrm{mEq} / \mathrm{L})$. The patient's BUN was $13.0 \mathrm{mg} / \mathrm{dL}$ and creatinine was $1.2 \mathrm{mg} / \mathrm{dL}$. Liver function tests were slightly elevated with an alkaline phosphatase of 179 IU/L, ALT of $79 \mathrm{IU} / \mathrm{L}$, and AST of $132 \mathrm{IU} / \mathrm{L}$. The patient had three sets of negative troponins. His lactate level was slightly elevated at $3.0 \mathrm{mg} / \mathrm{dL}$. The patient's coagulation studies were within normal limits.

Within the first forty-eight hours of hospital admission the patient's mental status improved towards baseline as noted by the patient's mother and girlfriend. On the second day of his hospital stay the patient's blood cultures from his first hospital admission in December were reported as positive for Mycobacterium avium intracellulare. That evening he was started on first line therapy for Mycobacterium avium intracellulare: clarithromycin $500 \mathrm{mg}$ by mouth every 12 hours, ethambutol $1600 \mathrm{mg}$ by mouth daily, and rifabutin $300 \mathrm{mg}$ by mouth daily.

An acute change in mental status was observed during the night of the fourth day following admission, two days after the patient had begun treatment for Mycobacterium avium intracellulare. He began to display disorganized thought processes and was unable to engage in meaningful conversation due to lack of insight. He was noted to have paranoid delusions, regarding the staff as being "evil" for purposefully attempting to disrupt his recovery by refusing to turn off the hospital's power. The patient would perseverate over the concept of electrical power, stating that he believed that the hospital's power was connected to him. At one point, when asked whether he had any suicidal ideations, he replied that he wanted to hurt himself by "turning on the light in the room." He also had delusions of grandeur, believing that he had the authority to fire medical staff whom he believed to be working for a local power company. At one point he stated, "I am going to infect the whole world with AIDS" and would often repeat that he "just gave the world AIDS." The patient exhibited inappropriately loud and pressured speech which progressed into severe thought blocking, resulting in the patient becoming nonverbal. During this time the patient was also noted to frequently open his mouth in a bizarre manner with no apparent stimuli.

On the day following the onset of psychotic symptoms and agitation neurology was consulted and they recommended electroencephalography which revealed diffuse background $2-4 \mathrm{~Hz}$ delta slowing, more prominent left hemispheric slowing, and few poorly formed sharp waves in the parietal/temporal region. Based on the results of the electroencephalography, the patient was started on levetiracetam by neurology for potential seizure activity. His acute psychosis and bizarre behavior continued for another day after the start of levetiracetam and at this point, psychiatry was consulted. Psychiatry noted that the altered mental status was of unclear etiology and recommended switching to an alternative antiepileptic since levetiracetam has been associated with increased agitation and psychosis. Psychiatry also recommended quetiapine at bedtime and as needed for agitation. After reviewing recommendations made by neurology and psychiatry it was decided that the patient should remain on levetiracetam and start the quetiapine regimen.

That same day a repeat cat scan of the head was done which showed no change from the initial image obtained in the emergency room. Lumbar puncture cerebrospinal fluid samples grew no organisms after seven days and only showed elevated protein at $75 \mathrm{mg} / \mathrm{dL}$. Infectious causes of delirium such as meningitis, toxoplasmosis, herpes simplex virus, cytomegalovirus, Epstein Barr virus, hepatitis $\mathrm{C}$, and John Cunningham virus were ruled out through examination of cerebrospinal fluid and MRI imaging. Syphilis was also ruled out through a negative VDRL serology. Metabolic causes such as B12 deficiency, folate deficiency, and thyroid abnormalities were also ruled out. HIV viral cultures from the cerebrospinal fluid were negative.

The patient's altered mental status continued for 3 more days despite the addition of quetiapine with no resolution of agitation or psychotic symptoms. Following consultation with infectious disease and clinical pharmacology, it was decided to substitute azithromycin for clarithromycin. Once clarithromycin was discontinued the patient's mental status returned to baseline within twenty-four hours and remained at baseline for the rest of his admission with no recurrence of psychotic symptoms or agitation. He was initiated on antiretroviral therapy a few days later and discharged with outpatient HIV follow-up

\section{Discussion}

Evaluation of delirium in a hospitalized patient requires a broad differential diagnosis including fluid/electrolyte imbalances, metabolic disorders, substance toxicity or withdrawal, infections, seizure, malignancy, and effects of medications. Drug toxicity accounts for approximately 30 percent of delirium cases [2]. The presentation of acute altered mental status in a patient with AIDS poses a unique challenge as additional considerations must be made of AIDS specific diagnoses such as opportunistic infections.

The severity of the patient's immune compromise rendered the importance of exploring the differential of opportunistic infections that contribute to neurocognitive disorders in HIV infected patients. Such conditions include JC virus encephalitis, cryptococcal meningitis, neurosyphillis, meningitis, CD8 encephalitis, and primary CNS malignancies such as CNS lymphoma. Latent reactivation of pathogens such as EBV, cytomegalovirus, and tuberculosis can also result in mental status abnormalities in the HIV-infected population. All of these conditions were ruled out in this patient through cerebrospinal fluid studies and brain imaging.

Lethargy and confusion are possible symptoms of metabolic disorders such as hyponatremia, an electrolyte disturbance usually caused by an inability to maintain proper water balance. Hyponatremia is a common electrolyte disorder found in hospitalized AIDS patients - it may be due to renal dysfunction directly caused by HIV 
(HIV-associated nephropathy) or SIADH, but is most likely to be multifactorial [3-5]. In our particular patient's case, hyponatremia was most likely secondary to diarrhea, which resolved over the course of his hospitalization. His hyponatremia was mild and unlikely to cause such profound neurologic symptoms. Other causes of delirium such as metabolic complications (B12/Folate deficiencies) and endocrine disorders (Hypo/Hyperthyroidism) were ruled out in this patient through laboratory testing.

Consideration was given as to whether the patient's altered mental status could be a direct result of the effect of HIV viremia on the brain. It has been previously shown that HIV coat protein gp120 increases intraneuronal calcium in vitro in a dose dependent manner [6]. It has therefore been postulated that manic symptoms may be caused directly by the HIV virus itself through this increase in intraneuronal calcium, a mechanism which has been implicated in the pathogenesis of bipolar disorder [7].

Prior to the advent of antiretroviral therapy HIV-associated neurocognitive disorder (HAND) was a significant cause of neurocognitive impairment including changes in mental status in AIDS patients. Antiretrovirals were developed that have higher CNSpenetration-effectiveness (CPE) resulting in a significant reduction in the incidence of HAND [8]. The CNS Penetration Effectiveness (CPE) score is a method to estimate CNS penetration of antiretroviral drugs. A CPE ranking of 1 indicates poor penetration and 4 indicates the best penetration. This method was developed using the CNS HIV Anti-Retroviral Therapy Effects Research (CHARTER) study, a multicenter, prospective observational cohort student based in six North American locations. Penetration of ART drugs is estimated based on CSF pharmacology, chemical characteristics and effectiveness in CNS infections [9]. Since our patient had not been started on any type of antiretroviral therapy prior to admission, HAND was initially considered among the differential diagnoses. Given that the patient's symptoms resolved without starting antiretroviral therapy or any other treatment for HAND, we concluded that an adverse effect to medication was a more probable source of delirium.

Clarithromycin, of the macrolide antibiotic class, has been suspected as the cause of a drug-induced delirium state with associated symptoms of psychosis or mania in a number of published case reports [10-18]. These cases have usually seen psychotic or manic symptoms following the use of clarithromycin for treatment of Helicobacter pylori induced peptic ulcer disease or respiratory infections. Although a direct mechanism by which clarithromycin may induce psychosis is unclear, macrolides have been found to inhibit glutamatergic neurotransmission, which is thought to contribute towards the pathologic mechanism of psychosis $[19,20]$. There are two cases of clarithromycin induced mania in patients with AIDS reported in a previous case report [21]. The authors, however, noted that both of their patients were underweight and receiving relatively high doses of clarithromycin. They also noted that since both patients had been taking fluconazole, an interaction between clarithromycin and fluconazole could not be ruled out. In our patient's case, a standard dose of $500 \mathrm{mg}$ of clarithromycin two times a day was administered. This was half of the dose received by the patients in the previous report. Additionally, our patient was not underweight and had not begun therapy with other potentially interacting drugs when he developed his symptoms.

\section{Conclusion}

In this patient's case, delirium accompanied by manic symptoms and psychosis began two days following initiation of ethambutol, rifabutin, and clarithromycin for the treatment of Mycobacterium avium intracellulare. His symptoms improved dramatically twenty-four hours following substitution of azithromycin for clarithromycin. His mental status subsequently remained stable for the remainder of his hospital admission. Based on the Naranjo nomogram, a scale used to quantify the likelihood of an adverse reaction being caused by a drug, the case received a score of 6 and thus it is probable that clarithromycin was the cause of this drug related adverse event [22]. We therefore recommend that clinicians consider clarithromycin as a possible cause of psychosis and mania and recommend azithromycin as an alternative treatment in patients exhibiting such symptoms after initiation of clarithromycin.

\section{References}

1. Coelho L, Veloso VG2, Grinsztejn B2, Luz PM2 (2014) Trends in overall opportunistic illnesses, Pneumocystis carinii pneumonia, cerebral toxoplasmosis and Mycobacterium avium complex incidence rates over the 30 years of the HIV epidemic: a systematic review. Braz J Infect Dis 18: 196-210.

2. Francis J (1996) Drug-Induced Delirium. CNS Drugs 5: 103-114.

3. Tang WW, Kaptein EM, Feinstein EI, Massry SG (1993) Hyponatremia in hospitalized patients with the acquired immunodeficiency syndrome (AIDS) and the AIDS-related complex. Am J Med 94: 169-174.

4. Vitting KE, Gardenswartz MH, Zabetakis PM, Tapper ML, Gleim GW, et al (1990) Frequency of hyponatremia and nonosmolar vasopressin release in the acquired immunodeficiency syndrome. JAMA 263: 973-978.

5. Glassock RJ, Cohen AH, Danovitch G, Parsa KP (1990) Human immunodeficiency virus (HIV) infection and the kidney. Ann Intern Med 112: 35-49.

6. Dreyer EB, Kaiser PK, Offermann JT, Lipton SA (1990) HIV-1 coat protein neurotoxicity prevented by calcium channel antagonists. Science 248: 364-367.

7. Kusumi I, Suzuki K, Koyama T (2012) [Cellular calcium signaling in bipolar disorder]. Seishin Shinkeigaku Zasshi 114: 821-828.

8. Cusini A, Vernazza PL, Yerly S, Decosterd LA, Ledergerber B, et al. (2013) Higher CNS penetration-effectiveness of long-term combination antiretroviral therapy is associated with better HIV-1 viral suppression in cerebrospinal fluid. $\mathrm{J}$ Acquir Immune Defic Syndr 62: 28-35.

9. Letendre S, Marquie-beck J, Capparelli E, Best B, Clifford D, et al. (2008) Validation of the CNS Penetration-Effectiveness rank for quantifying antiretroviral penetration into the central nervous system. Arch Neurol 65: 6570.

10. Shah M, Subhani M, Rizvon K, Mustacchia P (2012) Transient Psychotic Episode Induced by Helicobacter pylori Triple Therapy Treatment. Case Rep Gastroenterol 6: 381-386.

11. Feng Z, Huang J, Xu Y, Zhang M, Hu S (2013) Dissociative disorder induced by clarithromycin combined with rabeprazole in a patient with gastritis. J Int Med Res 41: 239-243.

12. Kouvelou E, Pourzitaki C, Aroni F, Papazisis G, Kouvelas D (2008) Acute psychosis induced by clarithromycin in a healthy adult? J Clin Psychopharmacol 28: $579-580$.

13. Lassnig RM (2010) [Acute psychosis induced by a Helicobacter pylori $(H$ pylori)-eradication treatment with amoxicillin, clarithromycin and pantoprazole] Neuropsychiatr 24: 144-150.

14. Htut Y, Kunanayagam S, Poi PJ (2006) Clarithromycin induced psychosis. Med J Malaysia 61: 263.

15. Warner A (2000) Clarithromycin--a precipitant for acute psychotic stress Psychosomatics 41: 539.

16. Neff NE, Kuo G (2002) Acute manic psychosis induced by triple therapy for $\mathrm{H}$. pylori. J Am Board Fam Pract 15: 66-68.

17. Nightingale SD1, Koster FT, Mertz GJ, Loss SD (1995) Clarithromycin-induced mania in two patients with AIDS. Clin Infect Dis 20: 1563-1564.

18. Pollak PT, Sketris IS, MacKenzie SL, Hewlett TJ (1995) Delirium probably induced by clarithromycin in a patient receiving fluoxetine. Ann Pharmacother 29: 486-488. 
Citation: Jacob S, Breig J, Chou T (2014) Clarithromycin Induced Psychosis in an AIDS Patient Receiving Treatment for Disseminated Mycobacterium avium Intracellulare Infection. J AIDS Clin Res 5: 297. doi:10.4172/2155-6113.1000297

Page 4 of 4

19. Manev H, Favaron M, Candeo P, Fadda E, Lipartiti M, et al. (1993) Macrolide antibiotics protect neurons in culture against the N-methyl-D-aspartate (NMDA) receptor-mediated toxicity of glutamate. Brain Res 624: 331-335.

20. Tuominen HJ, Tiihonen J, Wahlbeck K (2006) Glutamatergic drugs for schizophrenia. Cochrane Database Syst Rev: CD003730.
21. Nightingale SD, Koster FT, Mertz GJ, Loss SD (1995) Clarithromycin-induced mania in two patients with AIDS. Clin Infect Dis 20: 1563-1564.

22. Michel DJ, Knodel LC (1986) Comparison of three algorithms used to evaluate adverse drug reactions. Am J Hosp Pharm 43: 1709-1714. 